\title{
The Research of Clustering Algorithm for Wireless Ad Hoc Sensor
}

\author{
Jiuhua Zhang
}

\begin{abstract}
Stability in wireless ad hoc sensor networks cycle is one of the main evaluation parameters of the system. Network load is balanced and energy is in stable and sustainable is the important guarantee for the stability of the network. Traditional clustering algorithm for ad hoc network can effectively achieve a single point of network stability, and there is no efficient algorithm for long-term stability. Improvements have been made in this context traditional LEACH network clustering algorithm, based on efficient cluster head node using residual energy and communications networking sensor networks nodes remaining weaknesses in the energy networks and strengthening, improving the overall balance and effectiveness of the network, and ultimately to improve the long-term stability in wireless ad hoc networks sensor networks.
\end{abstract}

Keywords: Wireless Ad Hoc Networks · Sensor networks · Clustering algorithm node heterogeneous

\section{Introduction}

Many excellent characteristics for wireless ad hoc networks in the area of civilian and military traffic are occupying a place providing a favorable basis [1]. First, the network provides a cheap and rapid deployment of network may be organized. Second, jump and intermediate node forwarding characteristics can not change under the condition of network coverage, reduce the transmission power of each terminal, thereby reducing the antenna and the difficulty and costs related to sending and receiving parts of the design, thus contributing to the miniaturization of mobile terminals, provides low power consumption. From the perspective of sharing wireless channels, less low-power electromagnetic radiation generated by the radio waves, less impact on the health of users. In addition, network robustness

\author{
J. Zhang \\ Leshan Normal University, \\ Leshan, China \\ LSTCWD@163.com
}


and survivability to meet specific application needs. With the progress of the information society in wireless networks in people's lives play a ubiquitous communications carrier role. Various equipment for wireless network bandwidth and stability requirements continue to increase, and the current application in wireless ad hoc networks has had a breakthrough in technical difficulty, be models of largescale applications, but the inherent disadvantages of long-term stability and low so that it is becoming the focus of research in the field. Routing algorithms are important components of mobile ad-hoc networks; the algorithm of reliability issue has been plaguing the development a challenge. This highly reliable routing algorithm in wireless ad hoc sensor networks strategy as research, in-depth analysis of current policy and improved routing policies so that they have better security and resilience [2-5]. Study on routing algorithm for the amount of energy in new perspective, clustering and a series of technical improvements introduced.

\section{Algorithm in wireless ad hoc sensor network}

Wireless ad hoc network with no central structure, does not have absolute control in the Network Center. Equal status for all nodes, which is a peer network, the nodes through algorithms and distributed algorithm for hierarchical network coordinate their behavior. Nodes can join and leave the network at any time. Any node failure does not affect the entire network runs, compared with the network of canters, have strong survivability. Without Center and makes a wireless ad hoc network can achieve fast automatic network [6-9].

Wireless ad hoc network routing algorithm in accordance with the classification of different methods with different systems. Includes wireless routing algorithm (WRP), global link-state routing algorithm (GSR), dynamic source routing (DSR), on-demand distance vector routing algorithms (AODV), temporary routing algorithm (TORA), the destination sequence distance vector routing algorithms (DSDV).WRP (Wireless Routing Protocol) was introduced earlier, based on DBF algorithm on the basis of a table-driven routing algorithms. The WRP, each node needs to maintain the routing table information 4 tables: distance, routing table, the link cost table, packet retransmission lists. Node table of information is maintained through the acquisition of neighbor state changes, upon receipt of information from a neighbor, and saved to the table you want to change, and notify the adjacent nodes. If the node does not have a forward updated information, are sent regularly to the "HELLO" message to ensure the connectivity of nodes link. GSR (Global State Routing) algorithm, when a node receives a request message, first check the address of the requested information is present in the packet itself, if the packet is discarded, without your address written requests forwarded packets in the packet of information. Each node maintains the entire network topology information, and local optimization of routing information. DSR (Dynamic Source Routing) is a source-based routing for on-demand routing algorithms. 
Routing algorithm in wireless ad hoc networks as a problem that must be faced in the development of the technology, which there are many, but based on the current understanding of its development trends include the following. Future of secure routing in mobile ad-hoc network technology research should focus on the development of faster, more efficient secure routing algorithm with sufficient strength. Expects of its authentication mechanism is to compute short fast, keys, provided both source identification and anti-deny that simple and effective security mechanism, routing algorithm in mobile ad hoc network able to withstand attacks of false routing information. Secure routing algorithm for mobile ad hoc networks of formal analysis and certification of the correctness and security is also an important area of research. Long cycle routing algorithm in the overall development trend is the need to ensure the stability of the article.

\section{Improved wireless Ad hoc sensor network clustering algorithm}

Calculate consumption refers to the node in the consumption of energy for its operation. It mainly includes the CPU, memory, and physical devices, such as disk operation energy consumption. Address consumption refers to communicate with a wireless network interface in the consumption of energy. In ad-hoc networks, network interface can be in 4 different States: the launch, receive, idle and sleep a different state of the energy consumption of different. In hibernation, nodes do not consume energy; in the idle state, the node consumes a certain amount of energy; in the sending State, nodes use the transmitter to response and send a route request, route packets, and maximum energy consumption; In the receiving State, nodes use the receiver to accept the data, its energy consumption is also very large. In wireless ad hoc networks, link failure due to dynamic topology changes frequently, this requires routing algorithm in wireless ad hoc networks have to avoid infinite computations. So you need to make calculation of clustering algorithms to avoid infinite. Due to the limited wireless bandwidth in wireless ad hoc networks, transmission control group will consume some bandwidth, resulting in more limited available bandwidth. In order to make more efficient use of limited bandwidth resources, will require minimizing the control overhead. And because wireless mobile terminal in wireless ad hoc networks of CPU performance, memory size, external storage capacity, limited battery power, so you must try to save Terminal resources. Therefore, by increasing the complexity of the calculation for routing algorithms performance improvement is not applicable. Relatively simple routing algorithm helps to improve reliability and reduce cost, thus implementing routing features simplicity and routing algorithm in wireless ad hoc network design principle. In order to save the limited bandwidth, clustering algorithm for ad hoc network design principle is to minimize the amount of interaction between nodes, reducing the overhead of additional control information. Since Group network on broadcast channel of more jumped shared way will brings hidden Ter- 
minal, and exposed Terminal and intrusion Terminal, series of special of problem, cannot directly used existing of based on points on more points shared broadcast channel of MAC into clusters algorithm, needs design dedicated of MAC into clusters algorithm, as far as possible reduced group conflict to improve on limited channel of access efficiency, and balance on shared channel access of fair sexual problem. Portable terminal nodes in ad hoc network typically rely on battery power, limited transmitting power, requires node to the appropriate routing cluster algorithm to efficiently enable communication between the source and destination nodes. Since Group network routing into clusters algorithm design of target is quickly, and accurate and efficient, requirements in as possible short of time within found accurate available of routing information, and can adaptation network topology of quickly dynamic changes, while reduces introduced of additional delay and maintenance routing of control information, reduced routing into clusters algorithm of overhead, to meet moved Terminal calculation capacity, and storage space and power, area of limit, improve network of can extended capacity and more jumped routing of sound sexual. Currently most existing routing in ad hoc network clustering algorithm does not take into account the balanced distribution of routing between mobile nodes in the network tasks, makes clustering algorithm to run many of the results are routed through part of location at the Centre of a network of nodes. In this way, non-equilibrium distribution of data traffic will quickly run out of heavy load node of the electrical energy, as the power drain nodes increases, network connectivity will be weakened and there is a network of divisions, leading to route requests and data transfer fails, shorten the overall time to network. The routing on the energy consumption of the clustering algorithm is, to a large extent the clustering algorithm for load balancing problems in the design process, if this is good then the clustering algorithm with better adaptability. Loadbalanced routing is the use of packet transmission in nature there may be multiple paths to build group routing through a sufficient remaining capacity of grouped nodes forward to mitigate existing and potential local congestion, dynamic changes in the network load is transferred successfully. Evaluation of network performance indicator major throughput and network transmission delay, routing and load balancing technology's ability to improve both performance and can improve system throughput based on minimizing average packet transmission on the network end-to-end delay. This article in high reliability, low power routing cluster algorithm in the design of load balance based on the design, explored a network node-independent load balancing clustering algorithm for multi-channel.

In order to avoid algorithm for run-agnostic, the design assumptions in the channel status does not change during the appointment. In a conflict to avoid fallback mechanism of clustering algorithms, the assumption is clearly established. Nodes have a $\mathrm{m}(\mathrm{M}>\mathrm{l})$ available in channel network, select a channel as a whole network of common control channels for the delivery of public control of information, neighbors of node data channel status information exchange and free reservation, the rest of the M-1 channels as data channels. Each node maintains a neighbor node in the network status table, a table of channel State, recorded 
neighboring node availability and channel occupancy. Node of the data sent by the node's neighbor nodes reservation message state information into the channel in the RTS, and on common control channel broadcasting to the jump the neighbor node of the node. All neighbor nodes based on receiving the RTS message carried by the RTS information maintained by a neighboring node to this node and the availability of the data channels for dynamic updates. As the destination node's neighbor nodes after receiving the RTS, in addition to the neighbor node status table and maintained by channel using the above method updates the state table, also need to select a data channel to send and receive both sides are free to make an appointment. After receiving nodes receive RTS, maintained in addition to the neighbor node status and channel state table updates by the above method, you also need to choose to send and receive data channel that both sides are free to make appointments, its starting point is to try to keep network traffic in equilibrium on the data channel. Characterized the occupancy rate of the channel using channel load transfer and distribution of network traffic, you can channel usage fluctuations in mapping out business flow transmission on channel selection of the fair and balanced business stream in the data channel. Specific process of the algorithm is as shown in Figure 1.

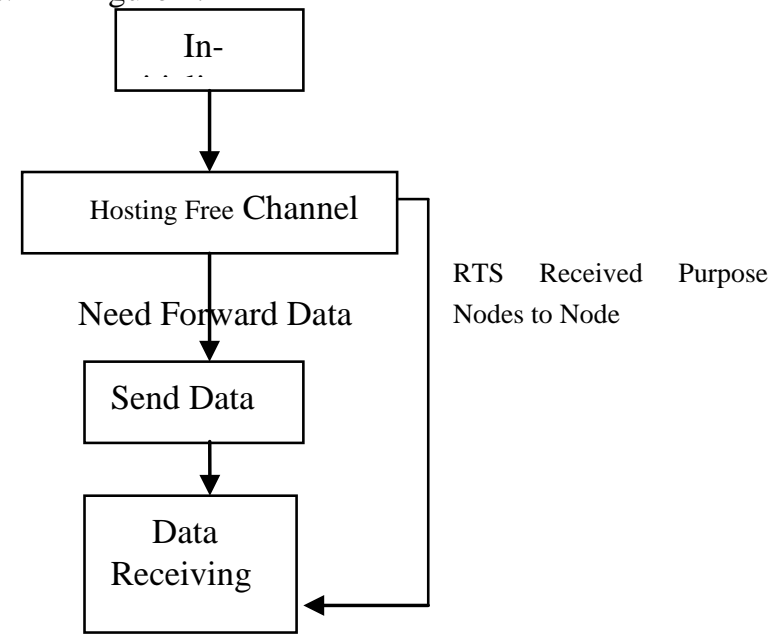

Fig. 1 Node-independent routing algorithm for clustering algorithms process flow.

\section{The new simulation of clustering algorithm}

This simulation uses simulation tools OPENET routing cluster algorithm for performance assessment. Specific parameters are set as: interconnection of all 60 nodes randomly, is $300 \mathrm{~m}$ the scope of the communication nodes, 256bits length groups controlled propagation delay for loss. Solstice=50us, FIFS_time=20us, 
DIF_TIME=120us. Packet arrival rate for the group for each node/speech common control channel and the data channel is set to $1 \mathrm{Mbps}$ bandwidth. Simulation of clustering algorithms in the evaluation focused on clustering algorithm for impact on network throughput and packet delay successfully sent. Because it is using a fully interconnected structure, simulation and statistics get the lower bound of the clustering algorithms. In the case of packet length is 1024bytes, BP to a balanced evaluation parameters and network traffic simulation of the relationship between the intensity and the number of channels. The specific packet arrival rate is as shown in Figure 2.

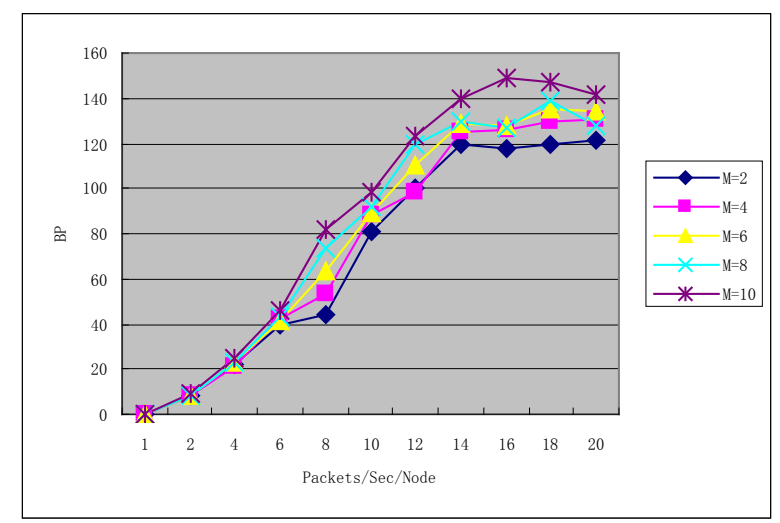

Fig. 2 A balanced evaluation parameters and grouping reach rate graph.

Clustering algorithm in this chapter can be seen from the figure under a different number of channels are able to adapt to changes in traffic intensity, for better load-balancing effect. Of course, on the performance evaluation index and method of load balancing are many, simple evaluation of traffic load on each channel of the multi-channel network is fully balanced distribution is one-sided. As business flow balancing the ultimate aim is to reduce the conflict and improve network packet sent in system throughput, extend battery usage time (extended network lifetime), traffic load balanced in a transmission is only a means to achieve network performance to improve. To comprehensive performance evaluation of load balancing, must also examine the network ultimately performance and throughput of packet transmission delay performance. As the packet arrival rate increase that traffic intensity increase. As can be seen from the diagram, with the growing number of channel $\mathrm{m}$, the throughput of the network is constantly growing. When the group arrived at the rate of common control channel throughput limit is reached, will cause more conflict control group, indicating that common control channel has been more effective data channels can no longer be assigned to a node, making the total throughput of the network growth slowed and eventually reaches a steady state. As can be seen from the diagram, in the case of small numbers of channels, by increasing the number of channels can be significantly improves the overall throughput of the network, when you increase the number of 
channels to a certain degree, simply increase the number of channels on the network's overall throughput improvement has been very limited.

Can be seen from the above figures compare proposed based on load balancing for multiple channels of wireless ad hoc network routing clustering algorithm with better than random selection of clustering algorithm for performance road access, its energy consumption is far lower than the current pandemic-routing cluster algorithm of random selection. And its long-term stability was also very good.

\section{Conclusions}

This article explores the current routing algorithm clustering algorithm for wireless ad hoc networks after the strengths and weaknesses of the algorithm has been improved. Low energy consumption is as the main design purpose of loadbalanced clustering algorithm for wireless ad hoc network routing, in particular dynamic load balancing on demand regardless of the nodes of roads by the clustering algorithm. The clustering algorithm in node when there is no data transfer channel resources used, number of channels required regardless of the maximum degree of connection and network, to support mobility of network nodes, low hardware requirements to implement clustering algorithm. In addition, the clustering algorithm in channel in the choice of appointment taking into account traffic loads between the different channels of distribution through the channel selection in a channel based on the occupancy rate to achieve a balanced distribution of business flow in channels, enabling clustering algorithm to adapt to the dynamic changes in the strength of network traffic and achieve increased overall network capacity. Found this article through simulation algorithms in sensor networks made by long-period performance stability is very prominent, merit promotion and further study.

\section{References}

1. R.Ramanujan,S.Kudige,S.Takkella,et al.Intrusion-resistant ad hoc wireless networks.IEEE Network,2002,2(7):890-894

2. Y-C Hu,A.Perrig,D.B.Johnson.Packet Leashes:A Defense against Wormhole Attacks in Wireless Ad Hoc Networks.IEEE Computer and Communications, 2003,3(30): 1976-1985

3. Q.Gu,P.Liu,C-H.Chu.Tactical bandwidth exhaustion in ad hoc networks.IEEE Information Assurance Workshop, 2004, 6(26): 3979-3983

4. Imad Aad,Jean-Pierre Hubaux,Edward W.Knightly.Denial of Service resilience in ad hoc networks.Mobile Computing and networking,2004,9(10):202-215.

5. ZHANG WEN-YA,L I ANG ZI-ZE.A power efficient routing protocol for wireless sensor network,Proceedings of the 2007 IEEE International Conference on Networking, Sensing and Control,Washington,DC:IEEE Computer Society, 2007: 20-25 
6. T.Thumthawatworn,P.Pakdeepinit.Method for cluster heads selection in wireless sensor networksProc.of the 2004 IEEE Aerospace Conference,Chiang Mai,2004:3615-3623

7. Murate T,Ishibuchi H.Performance evaluation of genetic algorithms for flowshop scheduling problems,In:Proc of the 1st IEEE Conf,on Evolutionary Computation. Orlando:IEEEPress, 1994:812-817.

8. KO R-S,LAI C-C. Density-based routing mechanism for sensor networks,Proceedings of the 10th IEEE Symposium on Computers and Communications,Washington, DC: IEEE Computer Society, 2005:341-346.

9. Chor Ping Low,Can Fang.Efficient Load_Balanced Clustering Algorithms for wireless sensor networks.Computer Communications,2008,31(3):750-759.

10. Ye M,Li C F.An energy efficient clustering scheme in wireless sensor networks.International Journal of Ad Hoc\&Sensor W ireless Networks, 2007, 3(2):99119.

11. Lian J,Naik K,Agnew G.Data capacity improvement of wireless sensor networks using non-uniform sensor distribution.International Journal of Distributed Sensor Networks,2006,2(02):121-145.

12. M.Ettus.System capacity,latency,and power consumption in multihop-routed SSCDMA wireless networks,in Proc.Radio and Wireless Conf,(RAWCON), Colorado Springs,CO,1998: 55-58. 